\title{
Der gerechte Frieden - zur Verortung eines Konzeptes
}

Semantisch grenzt sich der gerechte Frieden in zweifacher Weise ab: zum einen vom gerechten Krieg, zum anderen von einem ungerechten Frieden (vgl. hierzu auch Werkner, 2018, S. 35 ff.). Für die Entstehung des Konzeptes erweist sich die erste Abgrenzung - wie an obiger Stelle bereits beschrieben - als zentral. Eingeleitet wurde dieser Perspektivwechsel bereits mit der Ökumenischen Versammlung (1989, Ziff. 36):

\begin{abstract}
„Mit der notwendigen Überwindung der Institution des Krieges kommt auch die Lehre vom gerechten Krieg, durch welche die Kirchen den Krieg zu humanisieren hofften, an ein Ende. Daher muss schon jetzt eine Lehre vom gerechten Frieden entwickelt werden“.
\end{abstract}

Und auch nach dem ökumenischen Aufruf zum gerechten Frieden müsse „der Geist, die Logik und die Durchführung von Kriegen“ überwunden werden, womit auch die Lehre vom gerechten Krieg „obsolet“ werde (ÖRK 2011a, Ziff. 58). Da einige Kirchen gerade im angloamerikanischen Raum bis heute an der bellum iustum-Lehre festhalten und diese Formulierung nicht mittrugen, entstand im Weltkirchenrat die Kompromissformel: ,[Wir] gehen über die Lehre vom gerechten Krieg hinaus und bekennen uns zum gerechten Frieden" (ÖRK 2011b, S. 4). Aber auch im Raum der EKD gibt es Anfragen,

\footnotetext{
„ob nicht in dieser Perspektive eine Intention, die sich zumindest auch mit der Lehre vom gerechten Krieg verbindet, nämlich die Einhegung des Krieges durch die Mittel des Rechts, etwas zu wortreich verabschiedet wird - mit der Folge, dass diese Elemente über die Frage nach dem legitimen Einsatz rechtserhaltender Gewalt etwa in der EKD-Friedensdenkschrift von 2007 wieder eingeführt werden müssen“ (Anselm 2018, S. 54 f.).
} 
Beide Begriffe beziehungsweise Konzepte schließen sich per se erst einmal nicht aus. In diesem Kontext fordert auch Bernd Oberdorfer (2019, S. 17), „die plakative Gleichordnung der beiden Begriffe aufzugeben“, und plädiert für eine differenzierte Sicht auf beide Konzepte:

\begin{abstract}
„Der gerechte Frieden eignet sich als orientierender Leitbegriff für die friedensethische Diskussion. Aber im Horizont dieses Leitbegriffs kann und muss der Lehre vom gerechten Krieg (die die Funktion eines solchen umfassenden Leitbegriffs nie hatte!) eine - begrenzte, untergeordnete - Rolle eingeräumt werden. Dies dient der Transparenz der Argumentation und macht auch die Problemkontinuität sichtbar" (Oberdorfer 2019, S. 17).
\end{abstract}

Auch wenn der Leitbegriff des gerechten Friedens von seiner Genese her in Abgrenzung zur Lehre vom gerechten Krieg entstanden ist, impliziert er noch eine weitere Entgegensetzung: gerechter versus ungerechter Frieden. Und auch der letztgenannte Terminus mag - in ähnlicher Weise wie der Ausdruck des gerechten Krieges - auf den ersten Blick irritieren, drückt diese Wortkombination eines positiv konnotierten Begriffs mit einem negativ besetzten Attribut eine inhärente Spannung aus. Aber weder führt Ungerechtigkeit zwingend zum Krieg noch bringt Frieden notwendigerweise Gerechtigkeit mit sich. Im ersten Fall können entweder die Kosten eines Krieges, befürchtete Opfer und Schäden, den vorhersehbaren Nutzen übersteigen oder die Adressaten von Ungerechtigkeit greifen nicht zu militärischer Gewalt, da sie Krieg an sich ablehnen oder aber nicht über die entsprechenden Ressourcen verfügen, ihn aussichtsreich zu führen. Im zweiten Fall kann Frieden, verstanden als Abwesenheit von Krieg, auf ungerechten Verhältnissen wie Repression und Ausbeutung beruhen - ein Aspekt, der insbesondere für den globalen Süden von Relevanz ist.

Im Gegensatz dazu beansprucht der gerechte Frieden, mit seinem weiten und mehrdimensionalen Friedensverständnis über die auf Kriegsbegrenzung angelegte Lehre vom gerechten Krieg deutlich hinauszugehen, insofern es nunmehr gilt, die Bedingungen des Friedens zu bestimmen und herzustellen. Dabei bietet die inhaltliche Verknüpfung von Frieden und Gerechtigkeit den Ausgangspunkt für eine Kritik an bestehenden Gewaltverhältnissen, auch struktureller Art: „Soziale Gerechtigkeit tritt Privilegierungen entgegen, wirtschaftliche Gerechtigkeit dem Konsum und politische Gerechtigkeit Macht an sich" (ÖRK 2013a, S. 1). Insofern besteht zwischen Frieden und Gerechtigkeit „ein konstitutiver Zusammenhang“ (Brock 2017, S. 732).

Dieser Perspektivenwechsel impliziert aber zugleich eine prinzipielle, dem Konzept des gerechten Friedens inhärente Spannung: So gehen gute Dinge nicht immer zusammen (vgl. Müller 2013). Auch wenn Frieden und Gerechtigkeit 
normativ unauflösbar zusammengehören, können sie in der politischen Realität auseinanderfallen. Das ist immer dann der Fall, wenn Gewaltanwendung zum Schutz elementarer Menschenrechte alternativlos erscheint oder die Aufrechterhaltung des Friedens mit zentralen Gerechtigkeitsforderungen kollidiert. Und genau das bietet ,einen moralisch attraktiven Grund für Rebellion und Krieg und andere Formen kollektiver Gewalt" (Brock 2013, S. 23 f.). Diese Konsequenz ist auch aus westlicher Perspektive nicht unproblematisch, gelten gerade die humanitär begründeten militärischen Interventionen der letzten Jahre als wenig erfolgreich. Lässt sich diesbezüglich also eher mit Cicero entgegnen: „Ich, meines Orts, höre nicht auf, zum Frieden zu ermahnen: denn sogar ein ungerechter Friede ist nützlicher, als der gerechteste Krieg“ (in Wieland 1814, S. 90)?

Dieses Dilemma zeigt sich ebenso bei der Debatte um die internationale Schutzverantwortung, scheinen sich gerade hier das friedenspolitische Ziel der Gewaltminimierung und die Gewaltanwendung zum Schutz bedrohter Menschen angesichts schwerster Menschenrechtsverletzungen diametral gegenüberzustehen. Der Vorrang der Gewaltanwendung lässt sich widerspruchsfrei prinzipiell nur dann ethisch begründen, ,wenn Grundnormen der Gerechtigkeit dort, wo ihre Durchsetzung mit dem Ziel des Friedenserhalts in Konflikt gerät, als fundamentaler und ethisch dringlicher angesehen werden als der Verzicht auf Gewaltanwendung" (Hoppe und Werkner 2017, S. 353). Damit nicht jede Spannung zwischen Frieden und Gerechtigkeit zulasten der Friedensnorm geht, sind ethisch enge Grenzen notwendig. So kommt auch die internationale Schutzverantwortung ausschließlich bei schwersten Menschenrechtsverletzungen zum Tragen.

Die Spannung zwischen Frieden und Gerechtigkeit wird noch dadurch erschwert, dass sehr unterschiedliche Gerechtigkeitsvorstellungen existieren, die unter Umständen konträr zueinander stehen, zu Konflikten ausarten und Gewalt sogar noch befördern können. So ,[liegen] den meisten Kriegen und gewaltsam ausgetragenen Konflikten [...] miteinander konkurrierende oder sich gegenseitig ausschließende Gerechtigkeitsansprüche zugrunde" (Brock 2019, S. 119). Christopher Daase (2013, S. 173) identifiziert diesbezüglich fünf Arten von Gerechtigkeitskonflikten:

- Anwendungskonflikte infolge unterschiedlicher Sichtweisen über die Anwendung ein und desselben Prinzips und dessen Auswirkungen (beispielsweise bei der Verteilung materieller Ressourcen),

- Prinzipienkonflikte, die auftreten, wenn verfolgte Prinzipien in Widerspruch zu grundlegenden Gerechtigkeitsforderungen geraten (zum Beispiel wenn religiös motivierte Verteilungsforderungen der gleichberechtigten politischen Teilhabe von Frauen entgegenstehen), 
- Wertekonflikte zwischen gerechtigkeitsbezogenen und anderen - davon unabhängigen - gemeinwohlorientierten Ansprüchen (beispielsweise zwischen der im Rechtssystem etablierten retributiven, strafenden Gerechtigkeit und der auf Versöhnung und Konfliktdeeskalation ausgerichteten restaurativen Gerechtigkeit),

- Präferenzkonflikte, bei denen Gerechtigkeitsforderungen mit Eigeninteressen kollidieren (wie sie sich unter anderem bei Verhandlungen zum Klimaschutz zeigen) sowie

- Anerkennungskonflikte, die entstehen, wenn Gerechtigkeitsforderungen anderer prinzipiell nicht anerkannt beziehungsweise berücksichtigt werden.

So treten nicht nur Frieden und Gerechtigkeit zueinander in Spannung, sondern auch die verschiedenen Formen von Gerechtigkeitskonflikten können zu einer Quelle von Gewalt werden und sich hemmend auf den Frieden auswirken, ihn gegebenenfalls sogar gänzlich verhindern. Diesbezüglich setzt auch die EKD-Denkschrift auf Gerechtigkeitspostulate, die nicht unwidersprochen bleiben. So ist ihr eine spezifisch europäische - kritisch formuliert: eurozentristische Sichtweise zu eigen. Das zeigt sich beispielsweise an der sozio-ökonomischen Friedensdimension. Nach der EKD-Denkschrift (2007, Ziff. 83) erfordert der „Abbau von Not“ zweierlei: „Zum einen setzt er die Bewahrung der für menschliches Leben natürlichen Ressourcen voraus; zum anderen müssen Ungerechtigkeiten in der Verteilung materieller Güter und des Zugangs zu ihnen verringert werden.“ Danach lasse ,in den herrschenden systemischen Verhältnissen nur die Verteilung zu wünschen übrig“ (Duchrow 2019, S. 39). Versäumt wurde es - so die linksprotestantische Kritik von Ulrich Duchrow (2019, S. 39) -, ,eine systematische Analyse und Kritik der herrschenden politisch-ökonomischen Verhältnisse vorzunehmen, innerhalb derer die Friedensfrage im engeren Sinn erst zu verstehen sei.“ In diesem Kontext adressiert die EKD-Denkschrift auch kaum die Gewaltverhältnisse zwischen dem globalen Norden und dem globalen Süden; eine postkoloniale Perspektive auf Gerechtigkeitsfragen fehlt weitgehend mit konstitutiven Folgen:

\footnotetext{
„Wenn man [...] die Geschichte des Westens auch als eine Geschichte der Gewalt und der gewaltsamen kapitalistischen Zunutzemachung begreift, so wird deutlich, dass Begriffe wie ,Globalisierung ' und ,Wachstum` auch dazu dienen, diese gewaltvollen Praxen als Teil der westlichen Kultur zu verbergen“ (Betscher 2019, S. 94).
}

Die ökumenischen Debatten gehen hier deutlich weiter (vgl. Duchrow 2019). Der Ökumenische Rat der Kirchen beispielsweise erteilt dem imperialen Kapitalismus eine klare Absage: 
„Die wirtschaftliche Globalisierung hat den Gott des Lebens durch Mammon ersetzt, den Gott des freien Marktkapitalismus, der die Macht für sich beansprucht, die Welt durch die Anhäufung unmäßigen Reichtums und Wohlstands zu retten. [...] Aufgabe der Mission ist es somit, die Ökonomie der Habgier anzuprangern und die göttliche Ökonomie der Liebe, des Miteinanderteilens und der Gerechtigkeit zu praktizieren“ (ÖRK 2013b, Ziff. 108).

In grundlegender Kritik am Kapitalismus konstatiert auch Papst Franziskus in seinem Apostolischen Brief „Evangelii Gaudium“ im November 2013: „Diese Wirtschaft tötet" (Franziskus I. 2013; vgl. auch Segbers und Wiesgickl 2015). Mit ihrer Westzentrierung und Vernachlässigung der postkolonialen Perspektive bleibt die EKD-Denkschrift nicht nur hinter diesen Debatten zurück, sie steht damit auch dem eigenen Anspruch eines gerechten Friedens entgegen (vgl. Betscher 2019, S. 93). Die historischen und sozioökonomischen Kontexte kolonialer Strukturen und die in diesem Zusammenhang stehenden Machtformationen wirken bis heute fort. Sie reichen über ökonomische Gerechtigkeitsfragen hinaus und beeinflussen selbst Fragen kultureller Vielfalt und deren Anerkennungsverhältnisse, womit eine weitere Dimension des gerechten Friedens angesprochen ist. Aus postkolonialer Perspektive lasse sich die Kultur der Anderen eben ,nicht einfach abstrakt anerkennen“ (in Nachzeichnung der Betscher-Argumentation Munzinger 2019, S. 131). Angesichts fortbestehender Privilegien und asymmetrischer Machtverhältnisse seien die Voraussetzungen für eine „Anerkennung auf Augenhöhe“ (Betscher 2019, S. 92) häufig gar nicht gegeben, was die Kulturwissenschaftlerin Silke Betscher zu einer weiteren fundamentalen Kritik an der Friedensdenkschrift veranlasst:

„Das Diktum der Anerkennung und der Topos der ,kulturellen Vielfalt‘ $[\ldots]$ verstärken sich also (ungewollt) gegenseitig in der essentialistischen, binären und hierarchisierenden Konstruktion von Gesellschaften.“

Unabhängig davon, wie man sich zu dieser Kritik positioniert, verdeutlicht diese Debatte die Vielschichtigkeit und Komplexität des Gerechtigkeitsbegriffs und seiner Dimensionen und die Notwendigkeit, hier weiterzudenken. 
Open Access Dieses Kapitel wird unter der Creative Commons Namensnennung 4.0 International Lizenz (http://creativecommons.org/licenses/by/4.0/deed.de) veröffentlicht, welche die Nutzung, Vervielfältigung, Bearbeitung, Verbreitung und Wiedergabe in jeglichem Medium und Format erlaubt, sofern Sie den/die ursprünglichen Autor(en) und die Quelle ordnungsgemäß nennen, einen Link zur Creative Commons Lizenz beifügen und angeben, ob Änderungen vorgenommen wurden.

Die in diesem Kapitel enthaltenen Bilder und sonstiges Drittmaterial unterliegen ebenfalls der genannten Creative Commons Lizenz, sofern sich aus der Abbildungslegende nichts anderes ergibt. Sofern das betreffende Material nicht unter der genannten Creative Commons Lizenz steht und die betreffende Handlung nicht nach gesetzlichen Vorschriften erlaubt ist, ist für die oben aufgeführten Weiterverwendungen des Materials die Einwilligung des jeweiligen Rechteinhabers einzuholen. 